\title{
THE CALIFORNIA COLLOQUIUM
}

Differential Equations from the Algebraic Standpoint. By J. F. Ritt. New York, American Mathematical Society, 1932. x+172 pp.

The author sets himself the task of developing a theory of elimination which will reduce the existence problem for a finite or infinite system of algebraic differential equations to the application of the implicit function theorem taken with Cauchy's theorem in the ordinary case and Riquier's in the partial. The number of unknowns and independent variables is considered finite and fixed at the outset. The left members of the system are polynomials in the unknowns and their derivatives. The number of derivatives entering a given polynomial, presumably, is finite, though the number varies from polynomial to polynomial and may increase without limit in a sequence of polynomials.

The quest being, essentially, for holomorphic solutions of systems with holomorphic coefficients, every solution must satisfy what Riquier has named the prolonged system, which comprises all equations arising by differentiating the given equations. The author calls an algebraic differential system reducible or irreducible according as its prolonged system is algebraically reducible or not. One of the main currents of thought in the book seems to spring from the remark that an algebraically irreducible system may be reducible when regarded as a differential system.

The course followed by the author in accomplishing his purpose will now be indicated in summary fashion. It is shown that every system $\Sigma$ is equivalent to a finite number of closed irreducible systems

$$
\Sigma_{1}, \Sigma_{2}, \cdots, \Sigma_{s}
$$

in the sense that every solution of $\Sigma$ satisfies at least one $\Sigma_{i}$ and every solution of a $\Sigma_{i}$ satisfies $\Sigma$. For the case of a finite system, a theoretical process is developed $(\$ 67)$ for obtaining each of these irreducible systems in terms of a corresponding basic set $B_{\boldsymbol{i}}$, which contains at most one equation corresponding to each unknown and in addition has other special defining properties. A normal solution of $B_{i}$ is one for which none of a certain associated set of polynomials, called the separants, vanishes identically. Every solution of $\Sigma_{i}$ satisfies the corresponding $B_{i}$, and, in the nature of a partial converse, it is proved that every normal solution of $B_{i}$ satisfies the corresponding $\Sigma_{i}(\$ 66)$. Furthermore, every solution of $\Sigma_{i}$ which is not normal for $B_{i}$ satisfies a system $\Sigma_{i}$ which has a basic set $B_{i j}$ of lower rank than $B_{i}$. This makes it possible to replace (1) by a like finite set having the additional property that every solution of the original system $\Sigma$ is a normal solution for at least one of the basic sets which have been associated with the $\Sigma_{i}$.

The above, which applies literally only to the ordinary case, also describes the author's treatment of the partial case, if normal solution is replaced by regular, the latter type being one for which the set of non-vanishing polynomials has been augmented by the corresponding initials.

The final step is the development of a theoretical process for finding a finite set of equations equivalent to a closed irreducible system $\Sigma_{i}$. This is accom- 
plished by the extension of the Hilbert-Netto theorem to differential systems. As the author remarks, it remains to supplement the process by giving a means of specifying a priori an upper limit to the number of steps necessary in its application to a given system. The author determines a limit in the particular case of the closed irreducible system $\Sigma_{1}$ which gives the general solution for an algebraically irreducible equation of the first order in a single unknown and a single independent variable.

We turn now to a discussion of some details and by-products of the treatment. The elimination process employed is Euclid's division algorithm, which runs through the whole book, giving to the method a unity at times bordering on monotony. The reader is apt to wonder why the resolvent system for polynomials is not systematically employed. Its use in certain cases would be more efficient. For example, if one quadratic polynomial with literal coefficients is divided by another, the remainder is the resultant multiplied by an extraneous factor, which in the present terminology is the initial of the divisor (see Fricke, Algebra, vol. 1, p. 16). At the same time, this perhaps gives the reason why the division algorithm is not used in algebra to obtain the resultant.

As the author is of course aware (see his remarks on p. 62), there is a mixture of algebraic and function-theoretic methods in the book. Consequently the algebraic nature of a result is sometimes obscured by the analytic means of obtaining it. Related to this is the fact that the coefficients are taken from a certain field throughout. It is expedient to make thus assumption when an existence theorem is to be applied, but in the application of the division algorithm, for example, it is merely necessary that the coefficients belong to a ring which is closed under differentiation.

The author divides the unknowns into two sets, one of which he names "arbitrary." Names for both sets in keeping with the literature are principal and parametric. The two sets are characterized as follows: the system implies no equation free from principal unknowns, and implies, corresponding to each principal unknown, at least one equation containing it and no other principal unknown. In the ordinary case, let a set of principal unknowns be $y_{1}, \cdots, y_{p}$. There is a basic set $A_{1}, \cdots, A_{p}$ for the corresponding closed system such that each $A$ involves the $y$ with the same index and no $y$ with index higher than its own. An equation

$$
w-\lambda_{1} y_{1}-\cdots-\lambda_{p} y_{p}=0,
$$

linear in the principal unknowns and an auxiliary unknown $w$, is adjoined to the system. The augmented system has a basic set $R, B_{1}, \cdots, B_{p}$ having the same properties with respect to $w, y_{1}, \cdots, y_{p}$ as the $A$ 's with respect to $y_{1}, \cdots, y_{p}$. The circumstance that (2) is linear and of order zero has for effect that each $B$ is of order zero and linear in the $y$ of equal index. Consequently all the principal unknowns are expressible rationally in terms of the parametric unknowns, the auxiliary unknown, and their derivatives. The equation $R=0$ determines the auxiliary unknown $w$. It is called a resolvent of the system. Except for certain solutions of a special nature, the study of the original system reduces to that of the single equation $R=0$. The author takes pains to show that when the $\lambda$ 's are properly chosen functions of $x$, no two distinct solu- 
tions of the original system are associated with the same $w$. Other forms of (2) also lead to resolvents.

The application of the division algorithm requires that the polynomials be at least partially ordered relatively to one another. The scheme used is based on the lexicographical ordering, which is so prominent in Riquier's work. In order to avoid repetition (see pp. 3-6, 135, 142, 157-8) and to make the definitions clear and concise, placing a general discussion of this subject at the beginning of any treatment of systems would seem preferable to the author's way of introducing particular properties when and where they are needed. The essential features of the ordering are as follows. A set of symbols is called ordered if of any two symbols $x, y$ in the set it is possible to say whether $x$ precedes, coincides with, or follows $y$. These relations are conveniently denoted by $<,=,>$, and the terms positive and negative are useful in describing the relation of a symbol to 0 . If the symbols $x_{i}$ are taken from a set which is already ordered and which contains the difference of any two of its members, the symbols $x=\left(x_{1}, \cdots, x_{n}\right)$ can be ordered by saying that $x$ precedes, coincides with, or follows $y$ according as the first non-zero difference in the sequence $x_{1}-y_{1}, \cdots, x_{n}-y_{n}$ is negative, non-existent, or positive. The transitive law can readily be proved provided that two $x_{i}$ 's with the same sign have a sum with that sign. It is desirable at times to be able to compare $\left(x_{1}, \cdots, x_{n}\right)$ and $\left(y_{1}, \cdots, y_{m}\right)$, where $m<n$. Of the divers conventions that may be adopted we cite only two: $\left(y_{1}, \cdots, y_{m}\right)=\left(y_{1}, \cdots, y_{m}, 0, \cdots, 0\right)$ and $\left(y_{1}, \cdots, y_{m}\right)$ $=\left(y_{1}, \cdots, y_{m}, \infty, \cdots, \infty\right)$, where $\infty$ is a symbol with the properties $\infty-y>0$, $y-\infty<0, \infty-\infty=0, \infty+\infty>0$. The transitive law holds for both of these. We must use the second convention to order ascending sets in Ritt's manner (p. 4).

The symbols useful in the present work are obtained by starting from the non-negative integers $x_{i}$, forming from them hypercomplex integers $\left(x_{1}, \cdots, x_{n}\right)$, from the latter integers $\left(y_{1}, \cdots, y_{m}\right)$, where each $y_{i}$ is a hypercomplex integer, etc. The sets of symbols considered are such that the number of components in the parentheses is bounded. Any such set contains a minimum symbol. If it contains a maximum, it has only a finite number of distinct symbols (pp. 5, 6, 158). Each symbol represents a property of a polynomial, and the polynomial with the greater symbol has the property to a higher degree. Thus, the rank of a differential polynomial is the complex integer $(c, g, s)$, where $c$ is its class and $g, s$ are respectively its order and degree in $y_{c}$. The rank in a particular unknown $y$ is the complex integer $(p, q)$, where $p, q$ are respectively the order and degree in $y$. Something might be said in favor of sharpening the notion of rank so that any member of a basic set of a closed irreducible system would be algebraically irreducible. (See the author's repeated remark that a basic set can be chosen so as to have this property.)

It is interesting to compare the present method of elimination with Riquier's general method. In applying the latter, an equation has to be solved for one of the symbols in terms of the others, and the result substituted in a second equation. The fact that the eliminant is not in general expressed in finite terms gives rise to a difficulty, which is avoided by Ritt's method, whose eliminants appear as polynomials in the given functions and their derivatives. It is important, however, to realize that a difficulty of exactly the same nature may 
arise from the necessity of recognizing whether a given polynomial in the coefficients is identically zero. This difficulty will entirely disappear only if the coefficients are restricted to be polynomials in the independent variables.

Every one will recognize that the author's method has obvious advantages over Riquier's, but some will not agree with him when he says (footnote, p. 161) "It is not easy to imagine systems other than linear systems for which Tresse's argument and result have a definite meaning." The theorem and process in question, although often called Tresse's, are more appropriately ascribed to Riquier, who, in addition to supplying the convergence proof omitted by Tresse, published his result fully a year earlier. Contrary to the quoted statement, it seems quite easy to write down non-linear systems for which one is forced to admit that Riquier's method has a very definite meaning. The system consisting of an equation solvable in terms of radicals and any number of equations deduced from it by differentiation furnishes an example. No content of the general system is lost, moreover, if the word "solve" in the description of the method is given the somewhat obvious interpretation "determine all the roots." In the reviewer's opinion Riquier's result has a definite meaning even in the general case, and the criticisms to which it is open may be classed as practical rather than theoretical. This, of course, does not mean that further refinement of the method is impossible or not to be desired.

In conclusion, the book is the outstanding contribution to the theory of systems since the publication of Riquier's treatise. Unlike the latter, it is concise and readable. Original, precise, clear, stimulating, it seems to embody what the colloquium series should. The author and Society are to be congratulated on their achievement. 